\title{
Collaborative Load Balancing and Effective Channel Allocation for Cluster-Based MANETs
}

\author{
Sachin Gehlot, Meenu Vijarania, Swati Gupta
}

\begin{abstract}
Mobile ad hoc network is a wireless network group of mobile devices having no infrastructure. Load balancing is an important problem in such networks due to dynamic topology of the nodes. Many protocols are developed to improve the bandwidth, energy efficiency and throughput of these networks. With the increasing use of MANETs they are evolving wit use of applications and for non- uniform load distribution it's difficult to manage the load. Load balancing provides a single internet service from multiple servers as a commonly used application. Multicasting support at the link layer is essential for the efficient use of network resources. system propose a light weight dynamic channel allocation scheme for MANET. The system compares two algorithms DCA -TRACE and CMH-T RACE to provide support for non-uniform load distributions and propose CDCA-TRACE. It increases the number of source nodes and reduce the number of collisions, energy consumptions and average absolute IPDV drastically.
\end{abstract}

KEYWORDS- CSMA, Dynamic channel allocation, Load balancing, Mobile ad hoc networks

\section{INTRODUCTION}

Mobile Ad Hoc Network (MANET) It is a gathering of at least one association habitats with remote communication and network capacities that speak with one another without the assistance of a concentrated executive. It likewise has remote terminals that can powerfully network and send data without utilizing the current fixed network foundation. Directing channels are utilized to build up courses between center points to channel communications inside the network.

\section{Manuscript received May 22, 2020}

Sachin Gehlot, Department of Computer Science \& Engineering, Amity University, Gurugram, Haryana, India (email: sachingahlot96@gmail.com)

Meenu Vijarania, Assistant Professor, Department of Computer Science, Amity University, Gurugram, Haryana, India

Swati Gupta, Assistant Professor, Department of Computer Science, Amity University, Gurugram, Haryana, India
The essential objective of these specially appointed network steering protocols is to frame exact and proficient courses between sets of nodes with the goal that information is conveyed in an auspicious way. The development of the course should utilize a base utilization of overhead and transmission capacity. Portable nodes use distributed communication to shape networks and are called specially appointed networks.

Nodes in such networks are restricted by power, memory, transmission capacity, and computational requirements. Without any permanent infrastructure these networks have the ability to provide economical communication. MANETs are used [1] for military tactical operations, search and rescue missions, disaster relief, law enforcement etc.

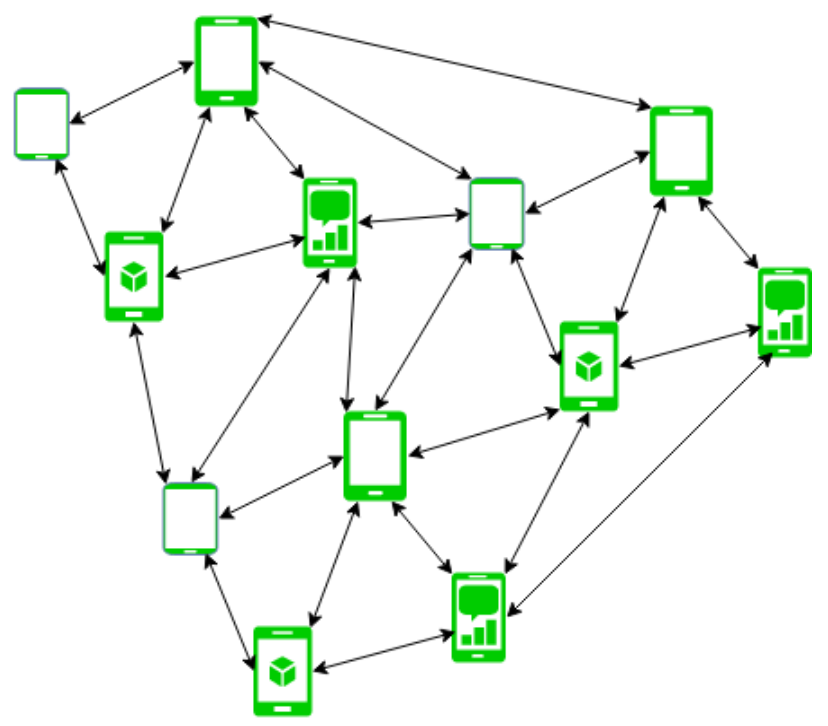

Fig 1: Mobile ad hoc networks

The proficient utilization of assets in versatile specially appointed networks (MANET) is essential to keep up the necessary nature of administration and expand the life of the network. Asset use, for example, transfer speed and force relies upon a few elements, for example, network size, hub thickness, and burden adjusting. These states are crazy and differ contingent upon network activities. The protocols that decide network conduct should powerfully adjust to these changing conditions to utilize assets proficiently. In this report, MANET's protocol engineering, which powerfully adjusts to changing conditions dependent on coordinated effort and data trade, prompts progressively 
effective utilization of framework assets contrasted with conflict-based models. Specifically, this record investigates the advantages of coordination-based adjustment and data trade in media get to control and network (steering) layers of the protocol stack. At the MAC layer, create scientific models that mirror the connection between protocol parameters and by and large protocol execution under different network conditions. This model shows that the parameters of the MAC layer protocol can be acclimated to boost channel assets, contingent upon application necessities and network conditions because of data trade, for example, normal network load thickness. A lightweight unique channel portion calculation that advances proficient utilization of assets dependent on nearby data trade to give a powerful framework that adjusts not exclusively to changing conditions yet in addition to spatially inconsistent traffic load adjusting AND is proposed an organized burden adjusting calculation. Broad reproductions show that both powerful channel distribution and facilitated load adjusting improve data transfer capacity proficiency under lopsided burden adjusting contrasted with protocols that don't utilize them The IEEE 802.11 system [16] and ungraceful protocol are looked at. Appropriate steering of information through MANET is another troublesome issue because of the dynamic conduct of the network, yet it is additionally significant regarding proficient utilization of assets. Two significant steering techniques, communicate and multicast, are explored over the network for pay and converged into a solitary system. The structure permits choosing the ideal steering plan dependent on the network conditions acquired through the trading of data, in this manner augmenting framework assets as far as range proficiency and vitality effectiveness. Moreover, association with different networks that exist together at a similar site as the network to a great extent decides its effectiveness. The advantageous networks approach with crossover nodes and the outcomes obviously show that harmonious networks can offer noteworthy help for co-found networks. This is particularly significant in networks with restricted assets like MANET. Hypothetical examination and reproduction are productive devices for looking at and assessing the efficiencies of different protocols, however these protocols are eminent for clock float, timing, defective physical layers, and obstruction from outside gadgets. It may not reflect numerous execution challenges. framework. Portable Ad Hoc Network is a self-arranging cell phone with no foundation. Every MANET hub goes about as a switch and can be sent whenever, anyplace. A few qualities of specially appointed versatile networks are dynamic topology, multi-jump steering with disseminated activity. Versatile nodes in the network move habitually and change topology. MANETs are getting progressively mainstream, and the normal network load considered in MANETs is expanding with the expanding number of utilizations. One of the principal issues with impromptu portable networks is the issue of burden adjusting. Burden adjusting is a strategy for disseminating the remaining task at hand among registering assets. Burden adjusting is the arrangement of a solitary Internet administration from different servers, an ordinarily utilized application. Burden adjusting is the fundamental field of versatile impromptu networks. Portable specially appointed networks are a significant class of networks and give communications backing to missions' Basic situations that incorporate war zones and strategic missions that expansion the significance of transmission capacity proficiency while keeping up tough force utilization deferral and jitter prerequisites. The tuned channel gets to protocol has been demonstrated to be reasonable for MANET with high burden and uniform burden conveyance. When all is said in done, in any case, these protocols are not reasonable for inconsistent burden adjusting as awkward channel get to protocols, as there is no unique on-request direct allotment instrument present in composed foundation-based protocols. It isn't ... Existing burden adjusting idea situations have numerous downsides. A portion of the drawbacks are that while sensor networks are progressively being executed in numerous application spaces, evaluating the unwavering quality of the information detailed by circulated sensors stays a troublesome issue. Sensors conveyed in brutal situations endure hub bargain Assaults by assailants attempting to embed bogus information into the framework. In this unique situation, assessing the dependability of the information gathered and introducing leaders about the unwavering quality of the information can be an overwhelming errand. Controlled by the emotional increment in registering intensity of ultra-low-power processors, driven basically by the interest for innovation plunges like portable figuring, WSN is equipment that can execute progressively refined trust accumulation and information assessment calculations. Will have the option to give. The ongoing appearance of multicore and multiprocessor frameworks in sensor nodes is a model. To defeat existing ideas, a portion of the ideas included are two calculations to address MANET lopsided burden offsetting in this report with this int Facilitated load adjusting calculations have been proposed in which the nodes pick a channel get to supplier dependent on range identification and asset accessibility.

\section{RELATED WORK}

MAC layer has a responsibility to coordinate the nodes and access to the shared radio channel for minimizing conflicts. In a multi-hop network, obtaining a high bandwidth efficiency is possible through the exploiting channel reuse opportunities. Since the early development stages of wireless communication, an efficient use of the common radio channel is the main attention.

Cidon et al. [13] presented a distributed dynamic channel allocation algorithm with zero optimality guarantees of a network with fixed a-priori controlled channel assignment. Alternatively, there are various theoretic approaches to this problem of channel allocation in MANETs. Gao et al. [11] modelled the channel allocation problem with multi-hop ad hoc wireless networks as a static cooperative game, in which few players collaborates to achieve a higher data rate. Due to the complexity of the optimal dynamic channel allocation these approaches are unscalable because it has been shown to be NP-hard. 
In a multi-hop wireless networks, CSMA techniques enables the same radio resources that is to be used in different locations which leads to increasing bandwidth efficiencies at the cost of possible collisions because of the hidden terminal problem. Different channel reservation techniques are used to tackle this problem. Karn et al. uses an RTS/CTS [12] packet exchange mechanism for the transmission of the data packet. It reduces the hidden node problem but unable to work under heavy network loads due to the exposed terminal problem. Several modifications are done to RTS/CTS mechanisms in order to increase the bandwidth efficiency including use of multiple channels.

However, these approaches attempt to solve the problem of channel assignment when there is a single destination of each transmission but do not cover group communication. In many cases, use of link layer multicasting/broadcasting increases the efficiency of network. In this paper, its focused-on link layer broadcasting with consideration of MANET scenarios where the destination of the generated packet is not a specific node in the local neighborhood but all the nodes in the immediate neighborhood of the transmitter. The IEEE 802.11 standards defines and allows link layer broadcasting services. In ad hoc broadcast communication mode, the IEEE 802.11 MAC DCF specification disables the RTS/CTS mechanism as well as ACKs. It is studied that for broadcast frames there is no MAC-level recovery or re-transmission.

In coordinated MAC protocols, channel coordinators do the channel assignment. Frequency reuse concept is used by them. Base stations form the basis of widely deployed GSM systems.

Two types of strategies are used for on-demand dynamic channel allocation which are centralized and distributed schemes. In a centralized DCA scheme, the available channels are kept in a pool and distributed to various cells by a central coordinator. It's effective in maximizing channel usage having high overhead and cannot be applied to MANETs because of the lack of high bandwidth and low latency links between the cluster heads for coordination.

In distributed DCA scheme, cellular networks are studied extensively. In it each cell is assigned with a number of channels which can be exchanged among adjacent cells through message exchange mechanisms between the channel regulators on basis of demand. This approach is also not applicable to MANETs. Unlike in cellular case, MANETs, exchanges messages between the channel regulators also consume network resources. Due to the node mobility and the dynamic topology of the network, having large overhead the bandwidth efficiency.

$\mathrm{Wu}$ et al. [10] extends the AODV protocol which includes a distributed system to infer the status of network and to optimize routes with the consideration of bandwidth efficiency and stability. A centralized load aware joint channel assignment and routing algorithm is proposed. Tseng et al. proposed a location aware DCA scheme for MANETs but it's necessary that location information is provided to each node.

In this paper, the concept and analyze the nonuniform load distribution problem is presented from the perspective of member nodes and the cluster heads. A collaborative load balancing algorithm for TRACE is also proposed. By combination of the dynamic channel allocation and collaborative load balancing algorithms, CDCA-TRACE protocol that has the highest bandwidth efficiency among the TRACE family of protocols is proposed. Performance investigation is done by comparing them to MH-TRACE, which implements the basic multi-hop MAC protocol of the TRACE system, as well as the beacon enabled IEEE 802.15.4 protocol in GTS mode of operation and the well-known IEEE 802.11 protocol.

\section{PROBLEM DEFINITION}

\section{A. Existing System}

The radio diverts considered in this report are described as broadband channels with low proliferation delay among source and goal sets contrasted with parcel transmission time. This recommends a third way to deal with utilizing channels. That is, Carrier Sense Multiple Access (CSMA) [22-23] mode. This plan attempts to maintain a strategic distance from crashes by identifying the bearer of another client's transmission. In view of this data about the condition of the channel, we can consider different activities performed by the terminal. Two protocols called "persevering" CSMA protocols are portrayed and examined. The protocols are depicted beneath, the essentials are portrayed, lastly the presentation of the exhibition delay is set and shown for every protocol. The different protocols considered underneath rely upon the activity taken by the terminal subsequent to distinguishing the channel. Nonetheless, in all cases, when the terminal discovers that the transmission fizzled, it reschedules the bundle for transmission as indicated by an arbitrarily circulated retransmission delay. At this new point, the transmitter recognizes the channel and rehashes the calculation determined by the protocol. A terminal that is prepared to send parcels is known as a prepared terminal. The terminal can impart or get signs, however not the two procedures simultaneously. Be that as it may, the deferral in changing starting with one mode then onto the next is immaterial. Moreover, the time required to recognize the bearer through parcel transmission is negligible (i.e., a zero-identification time is expected). All parcels are of consistent length and are sent through the normal commotion free channel. The framework accept that it isn't gotten (that is, if a portion of the two bundles cover, damaging impedance will happen and the two parcels should be retransmitted). The issue is additionally streamlined by expecting a similar spread deferral (little contrasted with parcel transmission time) for all source and goal sets. To start with, consider a non-relentless CSiWA. The thought here is to constrain impedance between bundles by continually reinventing parcels as they show up and the channel is known to be occupied. Two new MAC protocols have been characterized for specially appointed 
remote networks: dynamic opposite calculation (PBOA) and dynamic increasing speed calculation (PRUA). The two protocols isolate time into outlines with dispute spaces followed by information spaces. Contrasted with PBOA, PRUA doesn't perform power control and isn't as vitality productive as PBOA but it can achieve a transmission having tight packaging allowing nodes to make good decisions in contention period. For higher spatial reuse, contending nodes try to select potential destinations. Comparison is done between bot the protocols with carrier sense multiple access with collision avoidance (CSMA/CA), and also with the power control MAC (PCM) protocol. Also, there is a comparison between a hypothetical MAC protocol making optimal decisions and so achieve the network capacity. It is showed that both protocols perform better than CSMA/CA and PCM in terms of the throughput and robustness with respect to the choice of the routing protocol. Also, there is more energy efficiency in PRUA than CSMA/CA, and PBOA is more energy efficient than both CSMA/CA and PCM. However, due to their distributed nature, our protocols still cannot achieve the network capacity, even in simple network topologies. A new carrier-sense multiple access (CSMA) protocol is described for multihop wireless networks. The available bandwidth is divided into several channels and an idle channel is selected randomly for packet transmission in the CSMA protocol. The major difference is that there is no central infrastructure and thus the channel assignment is done in a distributed fashion via carrier sensing much as in a traditional CSMA scheme.

\section{PROPOSED SYSTEM}

For managing non-uniform load distribution in MANETs into the MH-TRACE framework two algorithms are used. Although MH-TRACE uses spatial reuse, it does not provide any channel borrowing or load balancing mechanisms and there is no optimal support for dynamically changing conditions and non-uniform loads. Hence, dynamic channel allocation and cooperative load balancing algorithms to MH-TRACE are used, creating new protocols namely, DCA-TRACE, CMH-TRACE and the combined CDCA-TRACE. CDCA-TRACE is a novel MAC protocol that maintains the same energy efficiency and channel regulation principles of MH-TRACE while enabling dynamic and scalable channel assignment of channels in addition to cooperative load balancing. Instead of message exchanges between the channel regulators (CHs), CDCA-TRACE utilizes spectrum sensing to keep track of channel usage in nearby clusters. It reduces the overhead found in dynamic channel allocation schemes for cellular networks and makes CDCA-TRACE suitable for MANETs. CDCA-TRACE also incorporates cooperation among the member nodes to improve the distribution of the load among the $\mathrm{CHs}$ and complements dynamic channel allocation to enhance the service rate. The contributions of this paper are:

i. We proposed a light weight dynamic channel allocation scheme for cluster-based MANETs.

ii. We proposed a collaborative load balancing algorithm iii. We have incorporated these two algorithms into the TRACE framework leading to DCA-TRACE and CMH-TRACE

iv. We have combined both algorithms leading to CDCA-TRACE that provides better support for non-uniform load distributions. We have compared the performance of these algorithms for varying network loads.

In order to overcome the difficulties, CMH-TRACE and CDCA-TRACE, which add cooperative $\mathrm{CH}$ monitoring and reselection on top of MH-TRACE and DCA-TRACE are proposed, respectively. In CMH-TRACE and CDCA-TRACE, nodes continuously look out the available data slots at the $\mathrm{CHs}$ around themselves announced by the beacon messages. When all the available data slots for a $\mathrm{CH}$ are allocated, with a probability, the active nodes attempt to trigger the cooperative load balancing algorithm. When the cooperative load balancing is triggered, the node that is currently using a data slot from the heavily loaded $\mathrm{CH}$ contends for data slots from other nearby $\mathrm{CHs}$ while keeping and using its reserved data slot until it secures a new data slot from another $\mathrm{CH}$. Size of the region over which the nodes are located, the number of nodes in the network, and their data generation patterns are all important in optimization of the design parameters. However, due to the dynamic topology of MANETs this information might not be available a priori, and some of these parameters may change during the network lifetime. Thus, it is necessary for the protocols to dynamically adjust to changing conditions. In uncoordinated MAC protocols such as IEEE 802.11, the common channel resource is shared among the nodes in the network based on carrier sensing. This simple behavior is well suited for handling any non-uniformities in the load distribution. However, these protocols do not scale well as the load in the network increases, due to the simple carrier sensing mechanism. On the other hand, coordinated MAC protocols such as the TRACE protocols are better suited for heavy load scenarios. Unlike MH-TRACE, the channel allocation for DCA-TRACE and CDCA-TRACE can be adjusted, making them more flexible protocols compared to their predecessor. By adjusting the channel access scheme, they are more capable of adapting to:

i. Shrinking network dimensions

ii. Non-uniformities in load distribution

Due to the movement of the nodes in the network, the diameter of the network may shrink over the course of network operation. At one extreme, when the largest distance between any two nodes in the network is below the communication radius, nodes form a single hop connected network. The bandwidth efficiency of MH-TRACE sharply reduces for such an operation, as MH-TRACE cannot adjust the number of frames in each superframe dynamically, and each $\mathrm{CH}$ can only utilize a single frame per superframe. However, the dynamic channel allocation mechanism of DCA-TRACE enables adaptation of the protocol to this environment by letting the single $\mathrm{CH}$ access all the frames and all the data slots. Thus, collaborative load balancing is ineffective for this scenario, and CDCA-TRACE operates similar to DCA-TRACE with the 
exception of reaction time. In CDCA-TRACE, CHs wait 3 super frames before accessing to an additional frame. This duration is smaller than the rate of increase in network load and hence does not alter the results. Confirming this, we observed same performance in both protocols and omitted the CDCA-TRACE results. For similar reasons, the CMH-TRACE results are also omitted. Due to the $\mathrm{CH}$ resignation mechanism in $\mathrm{MH}$-TRACE, only a single $\mathrm{CH}$ can operate in such a scenario. Since each $\mathrm{CH}$ only accesses one of the frames in each superframe and hence has access to only 6 data slots, the number of transmissions per superframe saturates at a value of 6 .

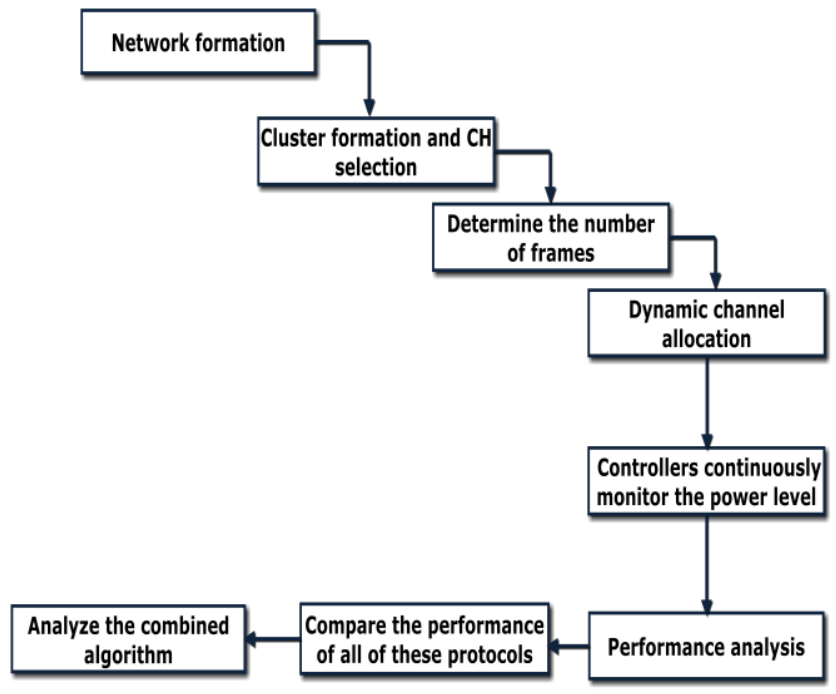

Fig 2: System Architecture

\section{Advantages:}

- The service levels are increased, shown to be very effective as well as the throughput in the system with minimal effect on energy consumption and packet delay variation.

- Maximized the improvements in the system.

- The proposed algorithm achieves a synchronization accuracy of $60 \mathrm{~ms}$ in this environment.

- It successfully runs an application providing real time voice communication.

- It also reduces the number of collisions, average energy consumption, and average absolute IPDV drastically, leading to a higher number of receptions and significant energy savings

\section{RESULTS AND CONCLUSION}

This area of report shows the results of the parameters such as packet delivery ratio, end to end delay and throughput after the execution of the schemes.

\section{A. Packet delivery ratio}

It is the ratio between the number of packets received with the number of packets that are sent.

$$
\text { Packet delivery ratio }=\frac{\sum \text { Number of packet receive }}{\sum \text { Number of packet send }}
$$

Below graph shows the PDR of the existing system with the proposed system.

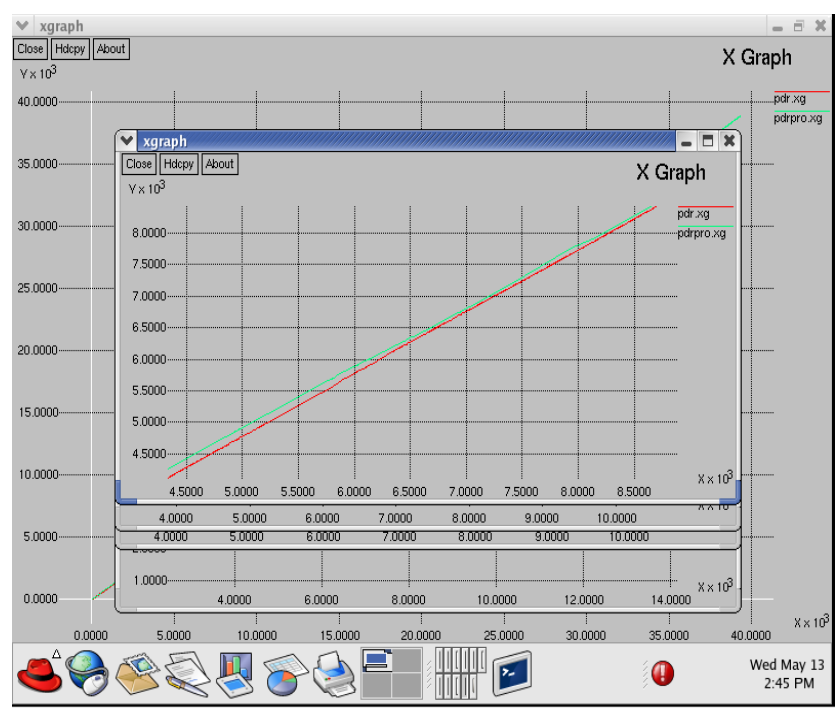

Fig 3: PDR variation of both the systems

\section{B. End to end delay}

It is the time taken by the packets to reach the destination from the source. It can be calculated by following formula and variation can be seen in the below graph.

\section{End to end delay $=\frac{\sum \text { Arrive time }- \text { Send time }}{\sum \text { Number of connections }}$}

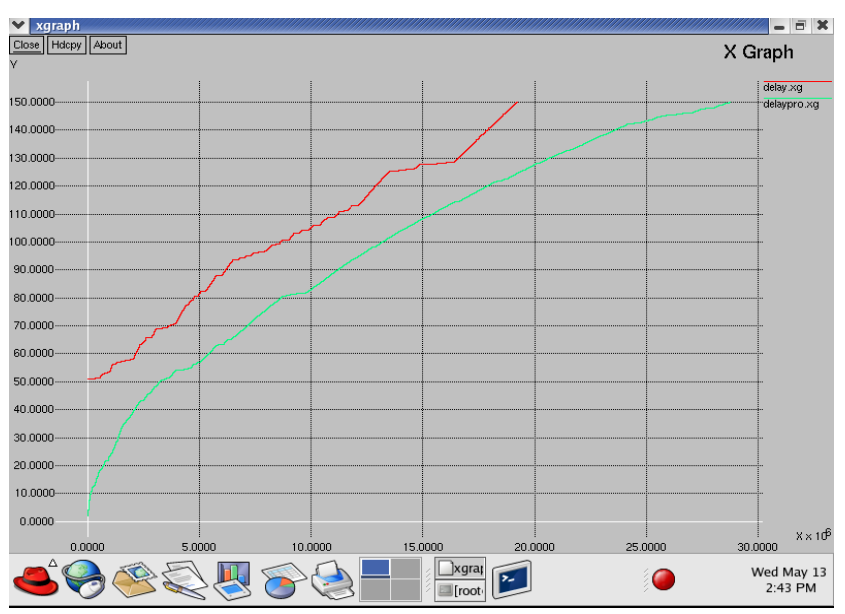

Fig 4: End to end delay variation in both the systems

\section{Throughput}

It is the ratio of the number packets received within a time period. With the increasing demand throughput increases. It can be calculated by the following formula and can be seen in below figure.

$$
\text { Throughput }=\frac{\sum \text { Number of packets received }}{\text { Time }}
$$




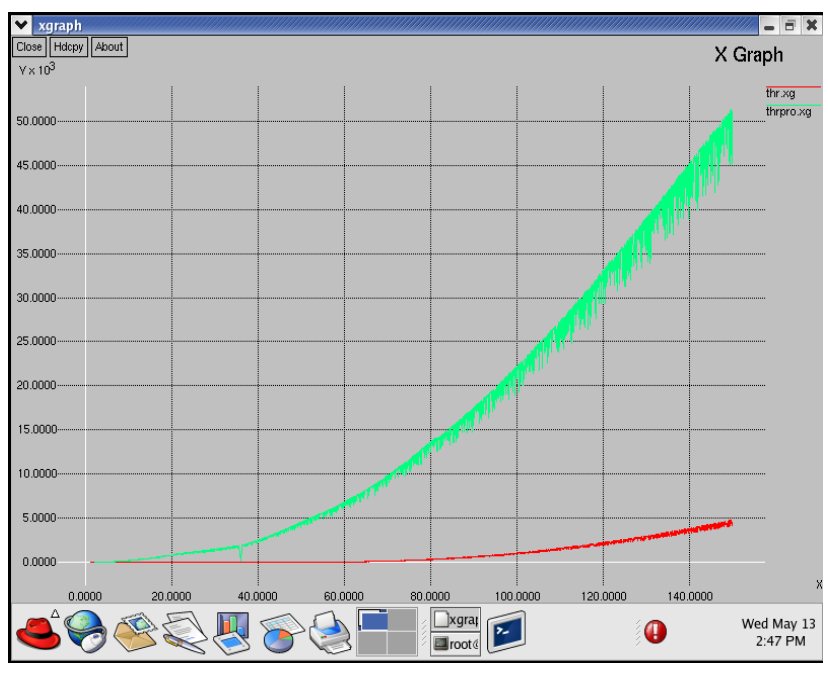

Fig 5: Throughput variations in both the systems

In the MANET field, vitality productivity is a significant issue here of research. To beat this issue, this report depicts a few calculations and protocols. On account of vitality productive MANET, different methodologies and examinations are being talked about to defeat the issue of vitality effectiveness. Picking the best way for information move is the directing procedure, and improving the exhibition of the framework's steering layer is the essential focal point of the examination. From source to goal, a directing based vitality cognizant pooling calculation is acquainted with structure different gatherings with ideal courses for sending bundles. You can see an expansion in the network life of the hub. Networks with bigger network sizes and more noteworthy portability require execution and adaptability to work better and all the more proficiently. All things considered; grouping is viewed as a significant research zone in versatile networks. Force utilization is additionally a significant issue in the MANET field as versatile nodes are battery controlled. With the assistance of the NS-2 test system, you can improve the parcel conveyance speed, start to finish delay and improved execution of the MANET versatile hub with the assistance of the Energy Aware Clustered Algorithm.

\section{REFERENCES}

[1] Jaichitra.I and Aishwarya.K, "A CDCA-TRACE MAC PROTOCOL FRAMEWORK IN MOBILE," International Journal of Future Innovative Science and Engineering Research (IJFISER), vol. 2, no. 2, p. 153.

[2] F. Tobagi and L. Kleinrock, "Packet switching in radio channels: Part II-the hidden terminal problem in carrier sense multiple-access and the busy-tone solution," IEEE Trans. Commun., vol. 23, no. 12, pp. 1417-1433, December 1975.

[3] B. Karaoglu and W. H. Tolga Numanoglu, "Analytical performance of soft clustering protocols," Elsevier, vol. 9, no. 4, pp. 635-651, 2011.

[4] J. L. R. Souza, A. Guerreiro and J. Runo, "Characterizing Inaccessibility in IEEE 802.15.4 Through Theoretical Models and Simulation Tools," INFORUM, 2012.
[5] B. Karaoglu and W. Heinzelman, "Multicasting vs. Broadcasting: What Are the Trade-Offs?," in IEEE Global Telecommunications Conference GLOBECOM 2010, 2010.

[6] S. V. Mary, J. P. Kumar, M. S. Victoria and K. Prathish, "Improving the energy efficiency in multicast routing for mobile adhoc networks," IEEE, 2013.

[7] "Installing Network Simulator 2 (NS2) on Ubuntu 14.04".

[8] "Wireless MAC and PHY specifications for Low-rate WAN networks," IEEE, vol. 15, no. 4, 2007.

[9] B. Tavli and W. B. Heinzelman, "MH-TRACE: Multi hop time reservation using adaptive control for energy efficiency," IEEE, vol. 22, no. 5, pp. 942-953, June 2004.

[10] M. Kappes, An experimental performance analysis of \{MAC \}ulticast in $802.11 \mathrm{~b}$ networks for VOIP traffic, vol. 29, 2006, pp. 938-948.

[11]L. Gao and X. Wang, "A game approach for multi-channel allocation in multi-hop wireless networks," in Mobile Ad hoc network computation, 2008, pp. 30-312.

[12]B. Leiner, D. Nielson and F. Tobagi, "Issues in packet radio net-work design," IEEE, vol. 75, no. 1, pp. 6-20, January 1987.

[13] G. Bhuvaneshwari and K. Vaitheki, "Load Balancing \& Channel Assignment for Cluster Based MANET in Heterogeneous Network," International Journal of Advanced Research in Electrical, Electronics and Instrumentation Engineerin, vol. 6, no. 1, March 2017.

[14] M. Felegyhazi, M. Cagalj, S. S. Bidokhti and J.-P. Hubaux, "Non-cooperative Multi-radio Channel Allocation in Wireless Networks," IEEE INFOCOM, 2007.

[15]X. Ma and X. Chen, "Performance analysis of IEEE 802.11 broad-cast scheme in ad hoc wireless LANs," IEEE Trans., vol. 57, no. 6, pp. 3757-3768, November 2008.

[16] J. Xie, A. Das, S. Nandi and A. Gupta, "Improving the reliability of ieee 802.11 broadcast scheme for multicasting in mobile ad hoc networks," in Proc. IEEE Wireless Commun. Netw. Conf., 2005.

[17] "Wireless LAN Medium Access Control (MAC) and Phys. Layer (PHY) Specifications," 2007.

[18]J. Zhu and S. Roy, "MAC for dedicated short range communications in intelligent transport system," IEEE Commun. Mag., vol. 41, no. 12, pp. 60-67, December 2003.

[19] K. Tang and M. Gerla, "MAC layer broadcast support in 802.11 wireless networks," in 21st Century Mil. Commun. COnf., 2000.

[20] J. So and N. H. Vaidya, "Multi-channel MAC for ad hoc networks: Handling multi-channel hidden terminals using a single transceiver," in Proc. 5th ACM Int. Symp. Mobile ad-hoc netw. comp., 2004.

[21]A. Nasipuri and S. Das, "Multichannel CSMA with signal power-based channel selection for multihop wireless networks," in Proc. 52nd Veh. Technol. Conf., 2000, 211-218.

[22] N. Jain, S. Das and A. Nasipuri, "A multichannel CSMA mac protocol with receiver-based channel 
selection for multihop wireless networks," in inProc. 10th Int. Conf. Comput. Commun. Netw, 2001.

[23] I. Shukla, L. Chandra and S. Iyer, "Mitigating the exposed node problem in IEEE 802.11 ad hoc networks," in Proc. 12th Int. Conf. Comput. Commun. Netw., 2003.

[24] A. Raniwala and T. Chiueh, "Architecture and algorithms for an IEEE 802.11-based multi-channel wireless mesh network," IEEE Comput. Commun., vol. 3, pp. 2223-2234, March 2005.

[25] A. Sen and M. Huson, "A new model for scheduling packet radio networks," in Proc. 15th Annu. Joint Conf. IEEE Comput. Soc. Netw. Next Generation, 1996.

[26]A.Ephremides and T. Truong, "Scheduling broadcasts in multi-hop radio networks," IEEE Trans. Commun., vol. 38, no. 4, pp. 456-460, April 1990.

[27] R. Ramaswami and K. Parhi, "Distributed scheduling of broadcasts in a radio network," in Proc. 8th Annu. Joint Conf. IEEE Com-put. Commun. Soc. Technol, 1989.

[28] L. Kleinrock and F. Tobagi, "Packet switching in radio channels: Part I-carrier sense multiple-access modes and their throughput-delay characteristics," vol. 23, no. 12 , pp. $1400-1416$.

[29]S. Toumpis and A. Goldsmith, "New media access protocols for wireless adhoc networks based on cross-layer principles," IEEE Trans. Wirelesse Communication, vol. 5, no. 8, pp. 2228-2241, Aug 2006.

[30]I. Cidon and M. Sidi, "Distributed assignment algorithms for multi-hop packet-radio networks," in 7th annual joint conference IEEE computation and communication, 1988. 